\title{
Immunization with dendritic cells pulsed ex vivo with recombinant chlamydial protease-like activity factor induces protective immunity against genital Chlamydia muridarum challenge
}

\section{Weidang $\mathrm{Li}^{1+}{ }^{\dagger}$,Ashlesh K. Murthy ${ }^{2 \dagger}$, B. K. R. Chaganty ${ }^{1}$, M. Neal Guentzel ${ }^{1}$, J. Seshu ${ }^{1}$, James P. Chambers ${ }^{1}$, Guangming Zhong ${ }^{3}$ and Bernard P. Arulanandam ${ }^{1}$ *}

' Department of Biology, South Texas Center for Emerging Infectious Diseases, University of Texas at San Antonio, San Antonio, TX, USA

${ }^{2}$ Department of Pathology, Midwestern University, Downers Grove, IL, USA

${ }^{3}$ Department of Microbiology and Immunology, University of Texas Health Science Center, San Antonio, TX, USA

\section{Edited by:}

Gregoire S. Lauvau, Albert Einstein

College of Medicine, USA

\section{Reviewed by:}

Timothy J. Sellati, Albany Medical College, USA

Nathalie Labrecque, University of Montreal, Canada

\section{*Correspondence:}

Bernard P. Arulanandam, South Texas Center for Emerging Infectious

Diseases, University of Texas at San Antonio, One UTSA Circle, San

Antonio, TX 78249, USA.

e-mail: bernard.arulanandam@

utsa.edu

${ }^{+}$Weidang Li and Ashlesh K. Murthy have contributed equally to this work.
We have shown that immunization with soluble recombinant chlamydial protease-like activity factor (rCPAF) and a T helper 1 type adjuvant can induce significantly enhanced bacterial clearance and protection against Chlamydia-induced pathological sequelae in the genital tract. In this study, we investigated the use of bone marrow derived dendritic cells (BMDCs) pulsed ex vivo with rCPAF $+\mathrm{CpG}$ in an adoptive subcutaneous immunization for the ability to induce protective immunity against genital chlamydial infection. We found that BMDCs pulsed with rCPAF + CpG efficiently up-regulated the expression of activation markers CD86, CD80, CD40, and major histocompatibility complex class II (MHC II), and secreted interleukin-12, but not IL-10 and IL-4. Mice adoptively immunized with rCPAF + CpG-pulsed BMDCs or UV-EB + CpG-pulsed BMDCs produced elevated levels of antigen-specific IFN- $\gamma$ and enhanced $\operatorname{lgG} 1$ and $\lg$ G2a antibodies. Moreover, mice immunized with rCPAF + CpG-pulsed BMDCs or UV-EB + CpG-pulsed BMDCs exhibited significantly reduced genital Chlamydia shedding, accelerated resolution of infection, and reduced oviduct pathology when compared to infected mock-immunized animals. These results suggest that adoptive subcutaneous immunization with ex vivo rCPAF-pulsed BMDCs is an effective approach, comparable to that induced by UV-EB-BMDCs, for inducing robust anti-Chlamydia immunity.

Keywords: Chlamydia trachomatis, dendritic cell, CPAF, UV-EB, genital infection

\section{INTRODUCTION}

Chlamydia trachomatis is an obligate intracellular Gram-negative bacterium that is the leading cause of bacterial sexually transmitted disease worldwide (Morrison and Caldwell, 2002; Gottlieb et al., 2010). Development of an effective vaccine has been suggested to be the most promising and effective strategy to prevent this disease (Rank, 1999; Brunham and Rey-Ladino, 2005; Hafner and McNeilly, 2008); however, an efficacious anti-chlamydial vaccine has yet to be licensed for human use. Current Chlamydia vaccine research efforts in general are focused mainly on the development of recombinant subunit vaccines including, but not limited to, the major outer membrane protein (MOMP; Pal et al., 2005; Kari et al., 2009; Sun et al., 2009), other outer membrane proteins (OMPs; Olsen et al., 2010), polymorphic membrane proteins (PMPs; Yu et al., 2009; Eko et al., 2011), a immunogenic plasmid protein (PGP3; Chen et al., 2010), and the protease-like activity factor (CPAF; Li et al., 2007, 2008; Murthy et al., 2007).

Some of these recombinant subunit candidates induce enhanced chlamydial clearance and reduced upper genital tract pathologies, but none of them induce sterilizing protective immunity. For example, we have shown previously that immunization with soluble recombinant ( $\mathrm{r}$ ) CPAF induces significantly enhanced genital chlamydial clearance (Li et al., 2010), reduction of oviduct pathology (Murthy et al., 2007), and preservation of fertility following primary and/or repeated chlamydial challenge (Murthy et al., 2011). The lack of sterilizing immunity suggested that either a single subunit is inadequate as a vaccine, or the need for a more effective delivery system to optimize the effect of a single subunit candidate. Dendritic cells (DCs) are professional antigen presenting cells (APCs) and key players in determining the type and quality of $\mathrm{T}$ cell immune response. Immature DCs in the peripheral tissue are highly active in phagocytosis and processing microbial antigens and undergo a maturation process as they migrate to draining lymphoid tissues. The utility of ex vivo antigen-pulsed DCs as an effective inducer of protective immunity has been extensively studied for tumor immunity and infectious agents (Svensson et al., 1997; Siegemund and Alber, 2008). To this end, ex vivo Chlamydia-pulsed dentritic cell (DC) transfer has been shown to induce robust protective immunity comparable to that induced by a live Chlamydia infection (Su et al., 1998).

In this study, we evaluated whether subcutaneous immunization with DCs pulsed ex vivo with soluble (r) chlamydial 
protease-like activity factor (rCPAF) would elicit an augmented immune response to induce sterilizing immunity against subsequent primary genital $C$. muridarum challenge. Bone marrow derived dendritic cells (BMDCs) pulsed ex vivo with rCPAF, with $\mathrm{CpG}$ as an adjuvant, displayed enhanced expression of activatory surface markers and secreted a high level of IL-12, an inducer of $\mathrm{T}$ helper 1 (Th1) type immunity. Mice immunized subcutaneously with the rCPAF-pulsed BMDCs induced a high level of antigen-specific IFN- $\gamma$ and anti-chlamydial antibody production, and induced significant protective immunity against genital Chlamydia infection.

\section{MATERIALS AND METHODS \\ RECOMBINANT PROTEINS, CHLAMYDIA, AND MICE}

The recombinant chlamydial proteins were purified as previously described (Li et al., 2008). Briefly, rCPAF constructs from C. trachomatis L2 genome with a 6X-Histidine tag (His) were cloned into pBAD vectors (Dong et al., 2004). The fusion proteins were expressed in Escherichia coli with L-arabinose as an inducer and extracted by bacterial lysis using sonication in a Triton X-100 lysis buffer. Ni-NTA agarose beads (Amersham, NJ, USA) were used for purification of rCPAF. The fusion protein was concentrated using Centriplus YM-10 tubes (Millipore, MA, USA), suspended in PBS with proteinase inhibitor cocktail (Roche, CA, USA), aliquoted, and then stored at $-20^{\circ} \mathrm{C}$. The purity of rCPAF was evaluated by SDS-polyacrylamide gel electrophoresis and by Western blot using antigen-specific murine antibodies. CpG (TCCATGACGTTCCTGACGTT) was obtained from Sigma Genosys (St. Louis, MO, USA).

Chlamydia muridarum was grown on confluent HeLa cell monolayers. Cells were lysed using a sonicator (Fisher Scientific, PA, USA) and elementary bodies (EBs) purified on Renograffin gradients as described previously (Li et al., 2007). Aliquots of bacteria were stored at $-70^{\circ} \mathrm{C}$ in sucrose-phosphate-glutamine (SPG) buffer.

Four to six week-old, female BALB/c mice were obtained from Charles River Laboratory (Bar Harbor, ME, USA). Mice were housed and bred at the University of Texas at San Antonio and provided food and water ad libitum. Animal care and experimental procedures were performed in compliance with the Institutional Animal Care and Use Committee (IACUC) guidelines.

\section{DENDRITIC CELL ISOLATION AND STIMULATION}

Bone marrow-derived dendritic cells were prepared as described (Su et al., 1998). In brief, 4-6 week-old female BALB/c mice were sacrificed and bone marrow was collected from both tibias and femurs. Erythrocytes were lysed by ammonium chloride treatment, and the cells immediately resuspended in Hank's Gelatin Buffer. After washing the cells with HBSS buffer, BMDCs were cultured in Iscove's Modified Dulbecco's Medium (IMDM) supplement with $10 \% \mathrm{FBS}, 4 \mathrm{mM} \mathrm{L}$-glutamine, $10 \mathrm{U} / \mathrm{ml}$ penicillin and streptomycin, $0.5 \mathrm{mM}$ 2-mercaptoethanol, $10 \mathrm{ng} / \mathrm{ml}$ each of IL4 , and granulocyte-macrophage-colony stimulating factor (GMCSF). On day 3, fresh $10 \mathrm{ng} / \mathrm{ml}$ each of IL-4 and GM-CSF was added to the culture. After 6 days of incubation, loosely adherent and non-adherent cells were harvested, washed, and resuspended in Hanks buffered salt solution (Gibco). A $2.3 \mathrm{ml}$ Opti-Prep concentrate (Sigma) was added to $9.7 \mathrm{ml}$ buffer [0.88\% (wt/vol) $\mathrm{NaCl}, 1 \mathrm{mM}$ EDTA, 0.5\% (wt/vol) bovine serum albumin, $10 \mathrm{mM}$ HEPES, pH 7.4, filter sterilized]. The cell suspension was gently layered on the top of $6 \mathrm{ml}$ of the Opti-Prep mixture and centrifuged at $600 \times g$ for $5 \mathrm{~min}$. The interface was collected and washed, and the cells cultured for another 1-2 days for use and the enriched DCs (over $85 \%$ purity as determined by CD11c surface expression using flow cytomtery) were used for experiments. For CpG, rCPAF + CpG, UV-EB + CpG stimulation, DCs were plated for $4 \mathrm{~h}$ in 24 -well plates at $10^{6}$ cells $/ \mathrm{ml}$ in the presence of either $10 \mu \mathrm{g} / \mathrm{ml} \mathrm{rCPAF}$ or UV-EB (at an MOI of 10) plus $30 \mu \mathrm{g} / \mathrm{ml} \mathrm{CpG}$, or with medium or $30 \mathrm{ug} / \mathrm{ml} \mathrm{CpG}$ alone; the cells were than washed and incubated for $24 \mathrm{~h}$. Cell supernatants were collected and stored at $-70^{\circ} \mathrm{C}$ for cytokine profiling. Analyses for IL-12, IL-10, and IL-4 in the supernatant were carried out by ELISA.

\section{FLUORESCENT ANTIBODY STAINING AND FLOW CYTOMETRY}

Dendritic cells alone or DCs pulsed with different antigens were stained with FITC, phycoerythrin, or APC-conjugated antibodies (CD80, CD86, CD40, MHC II, and CD11c). Staining was done in PBS containing 5\% FCS on ice for $20 \mathrm{~min}$, washed three times with PBS, and flow cytometry analysis was performed with an LSR II instrument (BD Biosciences).

\section{ANTIGEN-SPECIFIC CD4 ${ }^{+}$T CELL RESPONSES}

Fourteen days after mice had been previously challenged and resolved Chlamydia genital tract infection, spleens (3-5) were removed, and splenocytes layered over a density gradient to collect mononuclear cells. $\mathrm{CD} 4^{+} \mathrm{T}$ cell populations were isolated using negative selection magnetic particles (Stem Cell Technologies, BC, Canada) and the purity was determined to be at least $>90-95 \%$ of $\mathrm{CD}^{+} \mathrm{T}$ cells by flow cytometry using an PE-conjugated antiCD4 monoclonal antibody (BD Biosciences, CA, USA). A separate pool of naïve splenocytes was prepared from PBS (mock) immunized animals and treated with mitomycin-C (25 $\mu \mathrm{g} / 10^{7}$ cells $)$ for $20 \mathrm{~min}$ and used as a source of APCs. The purified CD4 ${ }^{+} \mathrm{T}$ cells $\left(5 \times 10^{5}\right.$ cells/well $)$ were cultured with APCs $\left(10^{5}\right.$ cells/well $)$ at a $5: 1$ ratio in $0.2 \mathrm{ml}$ and stimulated for $72 \mathrm{~h}$ in vitro with $\mathrm{rCPAF}$ $(2 \mu \mathrm{g} /$ well $), \mathrm{UV}$-inactivated C. muridarum $\left[10^{6}\right.$ inclusion forming units (IFU)/well, $10 \mathrm{MOI}$ ], the unrelated antigen hen egg lysozyme (HEL, $2 \mu \mathrm{g} /$ well), or medium alone in culture plates. Supernatants from the culture wells were analyzed for IFN- $\gamma$, IL-10, and IL-4 production using BD OptELISA kits (BD Pharmingen, NJ, USA) as described previously.

\section{ADOPTIVE IMMUNIZATION AND CHLAMYDIA CHALLENGE}

Dendritic cells were incubated with IMDM medium, rCPAF $\left(10 \mu \mathrm{g} / \mathrm{ml}\right.$ ), or UV-EB (ratio of $1: 10$ ) plus $30 \mu \mathrm{g} / \mathrm{ml} \mathrm{CpG}$ at $37^{\circ} \mathrm{C}$ for $4 \mathrm{~h}$, washed, and incubated in IMDM-10 containing GM-CSF for $24 \mathrm{~h}$. Then rCPAF + CpG or UV-EB + CpG-pulsed DCs were collected and injected subcutaneously into mice at a concentration of $1 \times 10^{6}$ cells/mouse in $0.1 \mathrm{ml}$ PBS twice at days 0 and 14. Seven days before Chlamydia challenge, mice were treated with $2.5 \mathrm{mg} / \mathrm{mouse}$ Depo-provera (Pfizer) to synchronize estrous. One month following the final vaccination, mice were challenged intravaginally with $5 \times 10^{4} \mathrm{CFU}$ of $C$. muridarum in $5 \mu \mathrm{l}$ of SPG 
buffer. Protection was assessed by quantifying the number of IFU recovered from vaginal swabs obtained on the indicated days after challenge. Entire genital tracts were removed from mice at day 80 after challenge and examined for the presence of hydrosalpinx, gross pathology evaluation, and histopathology.

\section{DETECTION OF ISOTYPE ANTIBODY LEVELS BY ELISA}

Ten days after the final immunization, sera from the animals were analyzed by ELISA as described previously (Li et al., 2007). Microtiter plates were coated overnight with $\operatorname{rCPAF}(5 \mu \mathrm{g} / \mathrm{ml})$ in sodium bicarbonate buffer $(\mathrm{pH} 9.5)$. Serial dilutions of sera were added to wells, followed by either goat anti-mouse IgG1 or IgG2a conjugated to alkaline phosphatase (Southern Biotechnology Associates, AL, USA). After washing, $p$-nitrophenyl phosphate substrate (Sigma, MO, USA) was added for color development and the absorbance at $405 \mathrm{~nm}$ measured using a $\mu$ Quant microplate reader (Biotek Instruments, VT, USA). Reciprocal serum dilutions corresponding to $50 \%$ maximal binding were used to obtain titers.

\section{VAGINAL C. MURIDARUM CHALLENGE AND DETERMINATION OF BACTERIAL SHEDDING}

One month following the final vaccination, animals were challenged intravaginally (i.vag.) with $5 \times 10^{4}$ IFU. Vaginal chlamydial shedding was determined on the indicated days for a period of 30 days following challenge, by swabbing the vaginas of infected mice and incubating the swabbed material on HeLa cell monolayers grown on glass cover slips in 24 -well plates. Cells were fixed with $2 \%$ paraformaldehyde and stained using a genus-specific rabbit monoclonal antibody and FITC labeled goat anti-rabbit secondary antibody. The number of inclusions was counted under a Zeiss Axioskop microscope, in 10 random mid-line fields for days 3-12 and over the entire cover slips for later time points. The mean \pm SEM of recovered chlamydial IFU per group of mice is reported.

\section{ASSESSMENT OF GROSS PATHOLOGY IN THE UPPER GENITAL TRACT AND HISTOPATHOLOGY}

Mice were euthanized at day 80 post-challenge, genital tracts collected, and the presence of gross hydrosalpinx was noted. The tissues were then photographed from a fixed distance at 6 megapixels using a Fuji F10 camera. Images were stored at high resolution and printed on sheets (A4 size) of paper. Dilatation of oviducts was scored as follows: the cross-sectional diameter of the oviducts was measured for the largest oviduct loop and the measurements for individual mice and mean $\pm \mathrm{SE}$ for each group was determined as described previously. Genital tracts also were examined for the presence of hydrosalpinx, then fixed in $10 \%$ neutral formalin, and embedded into paraffin blocks. Serial horizontal sections $(5 \mu \mathrm{m})$ were prepared and stained using hematoxylin and eosin (H\&E). Stained sections were visualized using a Zeiss Axioskop microscope and scored in blinded fashion as described previously. Cellular parameters [polymorphonuclear cells (PMNs), mononuclear, and plasma cells] were individually scored as follows: 0 , no significant presence of infiltration, 1 , presence of infiltration at a single focus, 2 , presence at two to four foci, 3, presence at more than four foci, or 4 , confluent infiltration. Results are expressed as mean \pm SD of scores from all animals in a group.

\section{STATISTICAL ANALYSES}

Sigma Stat (Systat Software Inc., San Jose, CA, USA) was used to perform all tests of significance. Student's $t$-test was used for comparisons between two groups and analysis of variance (ANOVA) between multiple groups for vaginal chlamydial shedding and dilatation of hydrosalpinx. A chi-square test was used to compare the incidence of hydrosalpinx. $p \leq 0.05$ was considered statistically significant. Microscopic oviduct pathology and histopathology scores were compared using ANOVA on ranks. All experiments were repeated at least twice, and each experiment was analyzed independently. In some experiments, where oviduct diameter data are shown as a composite of two experiments, the indicated significant difference holds true when the experiments are analyzed individually.

\section{RESULTS}

\section{BMDCs PULSED WITH rCPAF PLUS Cpg UP-REGULATED THE EXPRESSION OF SURFACE ACTIVATION MARKERS}

Bone marrow-derived DCs were generated and pulsed ex vivo with IMDM alone, CpG, rCPAF + CpG, or UV-EB + CpG. After $24 \mathrm{~h}$ incubation, BMDCs were collected, and then examined by FACS analysis for expression of surface activation markers CD86, CD40, CD80, and MHC II. The purity of CD11c ${ }^{+}$BMDCs was $85.3 \%$ (Figure 1A, right panel), which was consistent with previous reports of $82-86 \%$ (Eaton et al., 2006). Only $0.6 \%$ of the BMDCs stained with an isotype control antibody (Figure 1A, left panel) confirming the specificity of the anti-CD11c antibody. As shown in Figure 1B, BMDCs incubated with IMDM displayed a baseline expression of CD86 (64.8\%), CD80 (48\%), CD40 (27.8\%), and MHC II (65.2\%) and, as expected, the isotype control antibody stained cells displayed minimal expression of each marker. BMDCs incubated with CpG alone displayed enhanced expression of CD86 (83.4\%), CD80 (69.0\%), CD40 (80.4\%), and MHC II (90.1\%). BMDCs incubated with rCPAF + CpG or UV-EB + CpG also displayed enhanced expression of CD86 (80.9 or $79.6 \%$ ), CD80 (70.3 or $61.3 \%)$, CD40 (80.1 or $79.2 \%)$, and MHC II $(89.1$ or $86.1 \%)$. These results suggest that BMDCs pulsed ex vivo with the antigens, with $\mathrm{CpG}$ as an adjuvant, develop into a mature DC phenotype.

\section{BMDCs PULSED WITH rCPAF PLUS Cpg PRODUCE ELEVATED LEVELS OF IL-12}

Dendritic cells play an essential role in priming the differentiation of naïve T cells to Th1, Th2, or Th17 (Perry et al., 1997; Allam et al., 2009). Interleukin-12 is an especially potent inducer of Th1 immunity (Huang et al., 1999; Lu and Zhong, 1999), which has been shown to play a dominant role in protection against genital chlamydial infection (Zhang et al., 1999). Therefore, we evaluated the production of IL-12 by BMDCs pulsed ex vivo with $\mathrm{CpG}, \mathrm{rCPAF}+\mathrm{CpG}, \mathrm{UV}-\mathrm{EB}+\mathrm{CpG}$, or medium after incubation for $24 \mathrm{~h}$. The supernatants were assayed by ELISA for IL-12, IL-10, and IL-4 (Figure 2). All BMDCs displayed significantly $(p \leq 0.01)$ increased levels of IL-12, but not IL-10 or IL-4, when compared to BMDCs pulsed with medium alone. The levels of IL-12 production were enhanced comparably between the BMDCs treated with CpG $(1435 \pm 376 \mathrm{pg} / \mathrm{ml}), \mathrm{rCPAF}+\mathrm{CpG}(1028 \pm 63 \mathrm{pg} / \mathrm{ml})$, or UV-EB $+\mathrm{CpG}(1240 \pm 80 \mathrm{pg} / \mathrm{ml})$. These results suggest that 


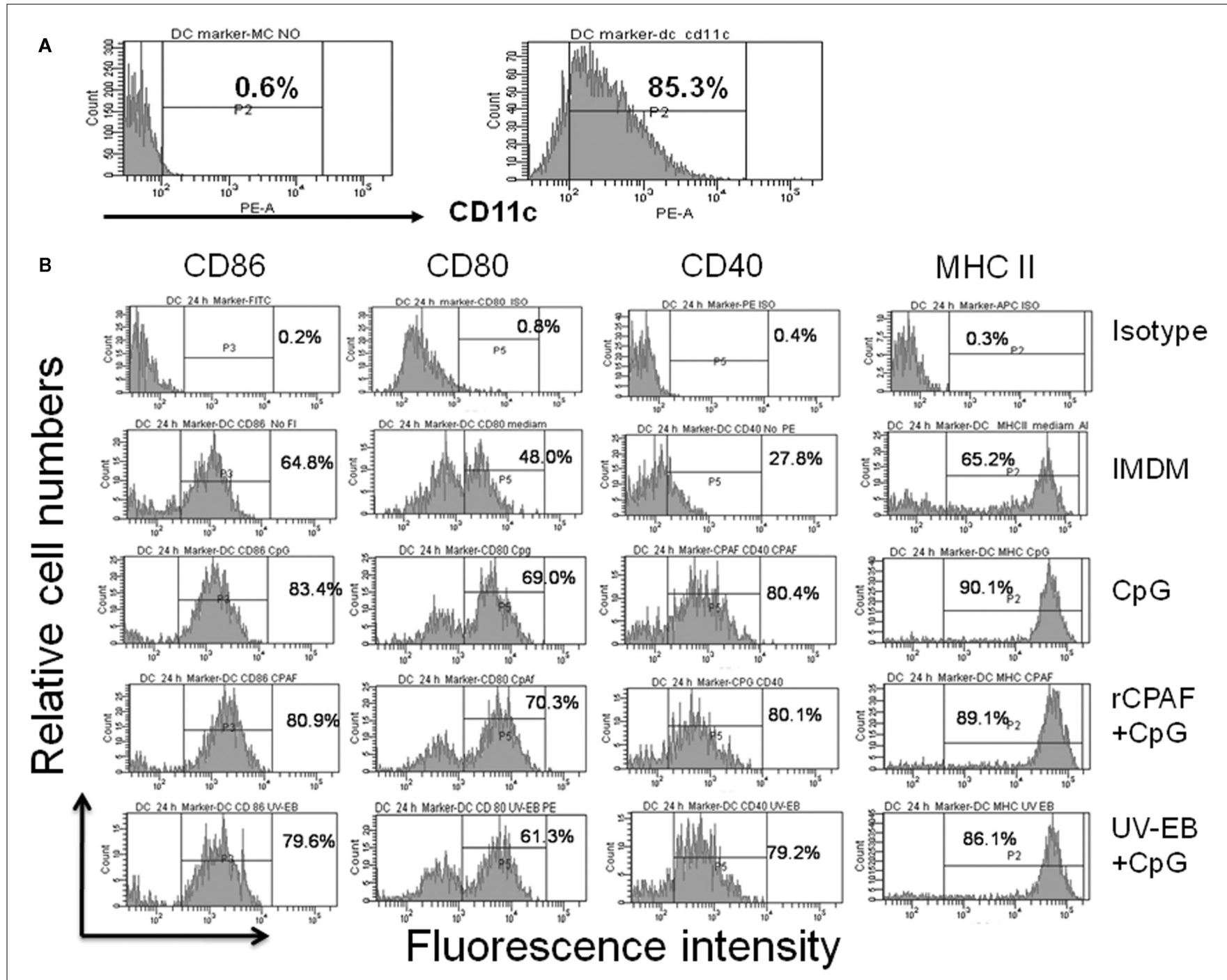

FIGURE 1 | Surface activation marker expression by BMDCs using FACS analysis. (A) The purity of BMDCs was determined by anti-CD11C antibody (B) Surface marker expression on BMDCs. Bone marrow-derived DCs were pulsed, washed, and cultured overnight as described in Section "Materials and Methods." Harvested DCs were surface stained for antibodies against CD86, CD80, CD40, and MHC II. The percentages of positive cells are indicated. One experiment from two independent experiments with similar results is shown.
rCPAF + CpG-pulsed BMDCs are capable of priming a robust Th1 response.

\section{BMDCs PULSED WITH rCPAF PLUS Cpg RESPOND TO INFECTION-SENSITIZED CD4 ${ }^{+}$T CELLS}

We also evaluated whether BMDCs pulsed ex vivo with rCPAF, UV$\mathrm{EB}$, or an unrelated antigen HEL were capable of processing and presenting antigens to elicit an antigen-specific IFN- $\gamma$ response from Chlamydia-specific CD ${ }^{+}{ }^{-} \mathrm{T}$ cells (Figure 3). BMDCs pulsed with $\mathrm{rCPAF}+\mathrm{CpG}$ and $\mathrm{UV}-\mathrm{EB}+\mathrm{CpG}$ induced Chlamydiaspecific $\mathrm{CD}^{+}{ }^{+} \mathrm{T}$ cells to produce significantly $(p \leq 0.01)$ higher levels of IFN- $\gamma(1194 \pm 309.5$ and $8298 \pm 586 \mathrm{pg} / \mathrm{ml}$, respectively $)$ compared to HEL pulsed BMDCs $(39 \pm 12.5 \mathrm{pg} / \mathrm{ml})$. As expected, naïve $\mathrm{CD}^{+}{ }^{+} \mathrm{T}$ cells displayed minimal IFN- $\gamma$ production upon culturing with BMDCs pulsed with the different antigens. These results suggest that BMDCs pulsed ex vivo with $\mathrm{rCPAF}+\mathrm{CpG}$ process and present CPAF epitopes to $\mathrm{CD} 4^{+} \mathrm{T}$ cells, leading to the production of high levels of IFN- $\gamma$ in an antigen-specific fashion.

\section{MICE IMMUNIZED SUBCUTANEOUSLY WITH EX VIVO ANTIGEN-PULSED BMDCS DISPLAY ROBUST ANTIGEN-SPECIFIC CYTOKINE AND ANTIBODY RESPONSES}

The induction of antigen-specific cytokine and antibody responses were evaluated following immunization with BMDCs pulsed ex vivo with $\mathrm{rCPAF}+\mathrm{CpG}$, UV-EB $+\mathrm{CpG}$, or $\mathrm{HEL}+\mathrm{CpG}$. Fourteen days following initial immunization, splenic $\mathrm{CD} 4^{+} \mathrm{T}$ cells were purified, cultured with mitomycin-C treated APCs, stimulated in vitro with rCPAF or UV-EB, and IFN- $\gamma$ production into the supernatant measured by ELISA. As shown in Figure 4A, mice vaccinated with $\mathrm{rCPAF}+\mathrm{CpG-BMDCs}$ exhibited significantly $(p \leq 0.05)$ greater rCPAF-specific IFN- $\gamma$ production $(1859 \pm 216 \mathrm{pg} / \mathrm{ml})$ than mice immunized with BMDCs 


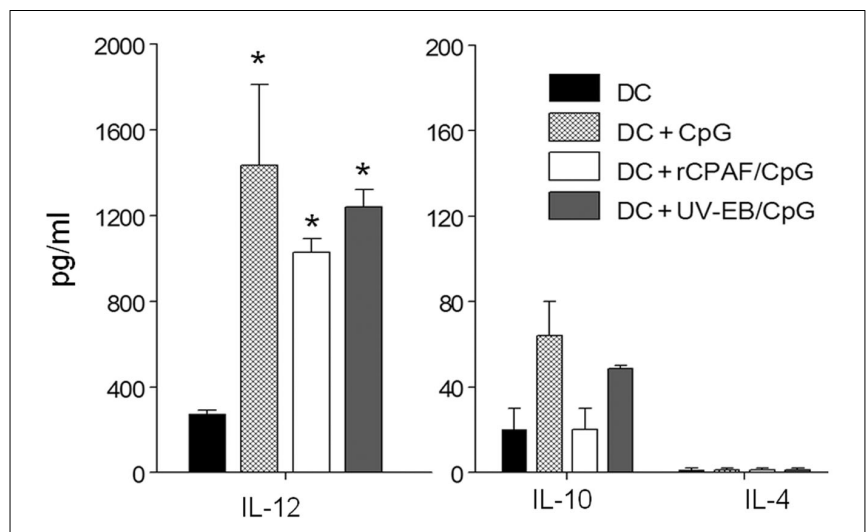

FIGURE 2 | BMDCs pulsed ex vivo with CpG, rCPAF + CpG,

UV-EB + CpG secrete IL-12. BMDCs were cultured in medium alone as a control or following pulsing with CpG, rCPAF + CpG, UV-EB + CpG for 4 h; the cells were than washed and incubated for $24 \mathrm{~h}$. Culture supernatants were collected and assayed by ELISA for IL-12, IL-10, and IL-4. The results are one of two independent experiments with similar results. $(*)$

Statistically significant differences ( $p<0.01$; ANOVA) between the indicated group and medium-pulsed BMDC are shown.

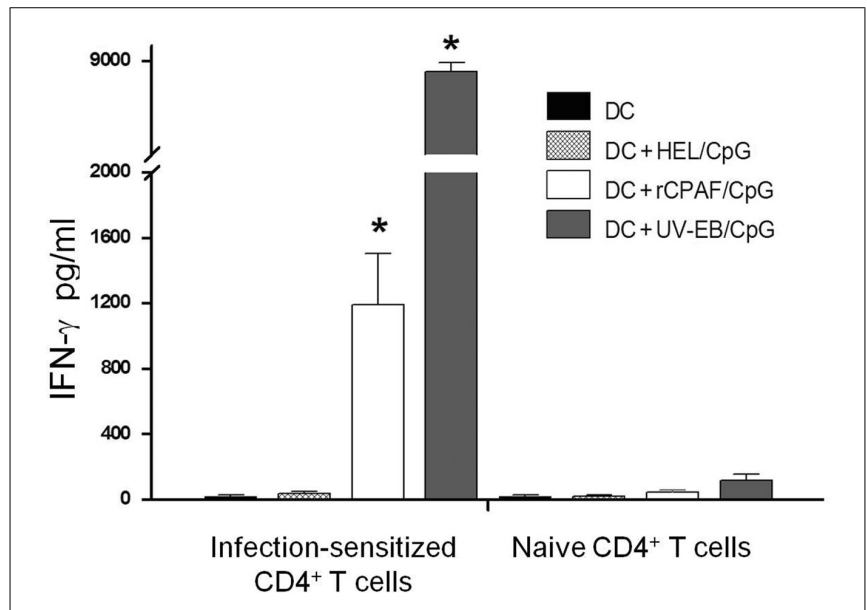

FIGURE 3 | Infection-sensitized CD4 ${ }^{+}$T cells secrete IFN- $\gamma$ in response to BMDCs pulsed with $\mathbf{r C P A F}+\mathbf{C p G}$ or $\mathbf{U V - E B}+\mathbf{C p G}$. CD4 ${ }^{+} T$ cells were collected from naïve mice or those mice $(n=3)$ that had resolved a Chlamydia genital infection. Purified $\mathrm{CD}^{+}{ }^{+} \mathrm{T}$ cells were incubated in the presence of unpulsed $B M D C$, and $B M D C$ pulsed with $\mathrm{HEL}+\mathrm{CpG}$, rCPAF $+\mathrm{CpG}$, or UV-EB $+\mathrm{CpG}$ for $72 \mathrm{~h}$, and supernatants was collected. IFN- $\gamma$ in supernatants was measured by ELISA. All experiment were performed in triplicate and repeated on two separate occasions with similar results. The results represent the mean \pm SEM from one representative experiment. (*) Statistically significant differences ( $p<0.01$; ANOVA) between the indicated group and $\mathrm{HEL}+\mathrm{CpG}$-pulsed BMDC are shown.

pulsed with UV-EB $+\mathrm{CpG}$ or $\mathrm{HEL}+\mathrm{CpG}$. As expected, purified $\mathrm{CD}^{+}{ }^{+} \mathrm{T}$ cells from animals vaccinated with BMDCs pulsed with UV-EB exhibited the greatest IFN- $\gamma$ production upon stimulation with UV-EB $(1953 \pm 119 \mathrm{pg} / \mathrm{ml})$. Purified CD4 ${ }^{+} \mathrm{T}^{-}$cells from animals immunized with BMDCs pulsed with HEL + CpG (mock immunized) displayed minimal IFN- $\gamma$ production upon stimulation with any chlamydial antigen, indicating the specificity of the measured cytokine responses. These results indicate BMDCs

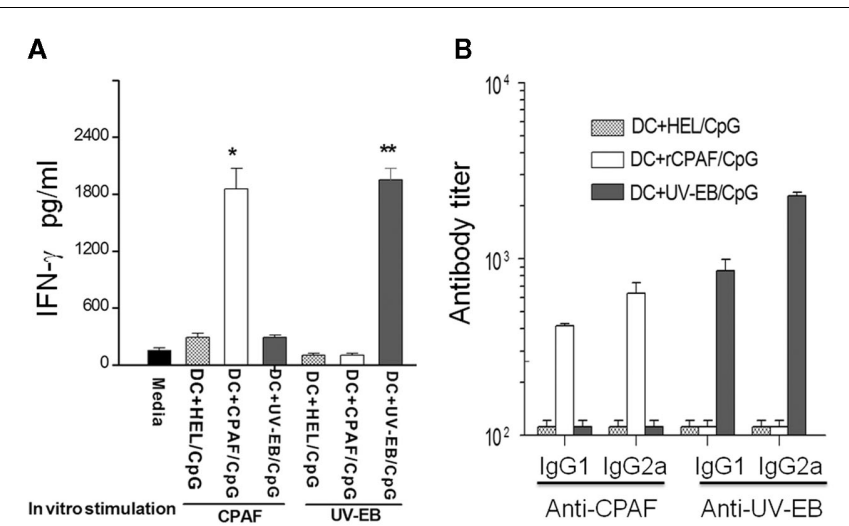

FIGURE 4 | Immune response following subcutaneous vaccination using BMDCs pulsed with HEL + CpG, rCPAF + CpG, UV-EB + CpG.

(A) Induction of antigen-specific IFN- $\gamma$. Groups (three mice per group) were immunized s.c. on day 0 with BMDCs pulsed with $\mathrm{HEL}+\mathrm{CpG}$, $\mathrm{rCPAF}+\mathrm{CpG}$, or UV-EB $+\mathrm{CpG}$. Fourteen days following initial immunization, splenic $\mathrm{CD} 4^{+} \mathrm{T}$ cells were purified, cultured with mitomycin-C treated APCs, stimulated in vitro with $\mathrm{CPPAF}$ or UV-EB, and IFN- $\gamma$ production into the supernatant measured by ELISA. The mean \pm SEM per group is shown. Significant differences ( $p \leq 0.05$; ANOVA) between the (*) CPAF $+C p G$ group and BMDCs pulsed with $\mathrm{HEL}+\mathrm{CpG}$ or UV-EB + CpG group, (**) between the UV-EB + CpG group and BMDCs pulsed with $\mathrm{HEL}+\mathrm{CpG}$, or $\mathrm{rCPAF}+\mathrm{CpG}$ group are shown. (B) The level of antigen-specific antibody. Groups (six mice per group) were immunized s.c. on day 0 with $B M D C$ s pulsed with $\mathrm{HEL}+\mathrm{CpG}, \mathrm{rCPAF}+\mathrm{CpG}, \mathrm{UV}-\mathrm{EB}+\mathrm{CpG}$ and booster immunizations were given on day 14 . The serum CPAF-specific and UV-EB-specific antibodies were measured 14 days after the last booster immunization, and the mean \pm SEM per group of the reciprocal titer corresponding to the $50 \%$ maximal binding is shown. The results are representative of two individual experiments.

pulsed with $\mathrm{rCPAF}+\mathrm{CpG}$ may elicit robust induction of antigenspecific IFN- $\gamma$ production in vivo.

The serum antibody responses against rCPAF and UV-EB were measured 14 days after the last booster immunization. As shown in Figure 4B, animals vaccinated with BMDCs pulsed with $\mathrm{rCPAF}+\mathrm{CpG}$ displayed elevated levels of anti-rCPAF IgG1 $(416 \pm 13.8)$ and IgG2a (639 \pm 95.1$)$ antibody. As expected, animals immunized with BMDCs pulsed with UV-EB + CpG exhibited high levels of anti-UV-EB IgG1 $(858 \pm 136.3)$ and IgG2a (2274 \pm 111.5$)$ antibody. Animals immunized with BMDCs pulsed with HEL + CpG (mock immunized) exhibited minimal anti-rCPAF and anti-UV-EB antibodies. Collectively, mice immunized with BMDCs pulsed with rCPAF displayed elevated levels of antigen-specific IFN- $\gamma$ production and increased levels of antirCPAF IgG2a antibodies, suggesting the induction of a robust Th1 type response.

\section{IMMUNIZATION OF MICE WITH BMDCs PULSED WITH rCPAF PLUS CpG ENHANCES THE RESOLUTION OF GENITAL C. MURIDARUM INFECTION}

To determine the protective immunity induced by immunization with rCPAF-pulsed BMDCs, immunized mice were challenged intravaginally with C. muridarum 1 month after the second adoptive immunization. Vaginal chlamydial recovery was measured every third day post-challenge for a period of 30 days, vaccination with DC + HEL/CpG (unrelated antigen) served as the baseline 
for the degree of Chlamydia shedding, which was similar to mockvaccinated mice over a 30-day period. As shown in Figure 5A,B, vaccination with rCPAF-pulsed BMDCs induced a significant reduction $(p<0.05)$ in bacterial shedding, with clearance of the infection in $50 \%$ of the mice as early as day 15 , and in all (100\%) mice by day 18 after challenge. In comparison, all of the HELBMDC (mock) immunized animals were still shedding Chlamydia on day 18 after challenge, and exhibited complete clearance on day 30 after challenge. As expected, animals immunized with UVEB pulsed BMDCs began to resolve the infection as early as day 3 after challenge, and all (100\%) mice in this group completely cleared the infection by day 18 after challenge. These results suggested vaccination with BMDCs pulsed with $\mathrm{rCPAF}+\mathrm{CpG}$ did not induce resistance to infection, but mediated significantly accelerated chlamydial clearance leading to complete resolution of infection within the same time period as UV-EB pulsed BMDC immunized animals.

\section{IMMUNIZATION OF MICE WITH BMDCS PULSED WITH RCPAF PLUS CPG INDUCES PROTECTION AGAINST C. MURIDARUM-INDUCED UPPER GENITAL TRACT PATHOLOGY}

To determine the effect of immunization with rCPAF-pulsed BMDCs on development of Chlamydia-induced disease sequelae, the development of hydrosalpinx on day 80 after challenge was evaluated. Vaccination with BMDCs pulsed with rCPAF + CpG reduced the development of hydrosalpinx (17\% bilateral) on

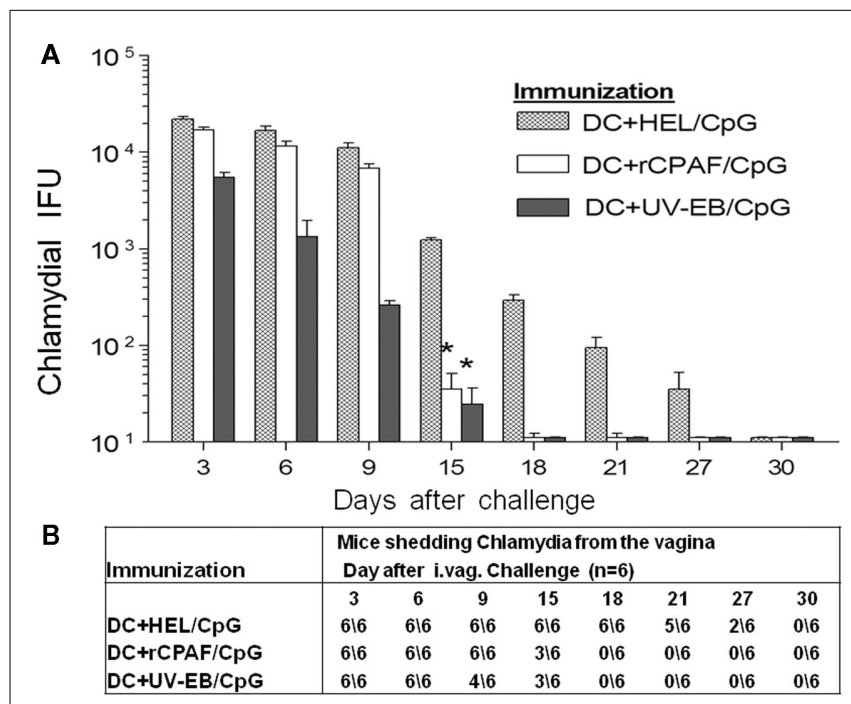

FIGURE 5 | (A) Vaginal chlamydial shedding was measured post-challenge. Groups (six mice per group) were immunized s.c. with BMDCs pulsed with $\mathrm{HEL}+\mathrm{CpG}, \mathrm{rCPAF}+\mathrm{CpG}$, or UV-EB + CpG, and booster immunizations were given on day 14 . One month after the final immunization, mice were challenged i.vag. with $5 \times 10^{4} \mathrm{IFU}$ of C. muridarum. Chlamydial shedding was monitored every third day after challenge for 1 month, vaccination with $\mathrm{DC}+\mathrm{HEL} / \mathrm{CpG}$ (unrelated antigen) served as the baseline for the degree of Chlamydia shedding, which was similar to mock-vaccinated mice over a 30-day period. The mean \pm SEM of chlamydial shedding per group at each time point is shown. $(*)$ Significant reductions $(p<0.05$; one-way ANOVA) in bacterial shedding at the indicated time points between the indicated group and HEL + CpG-pulsed groups are shown. The results are one experiment from two independent experiments with similar results. (B) The number of animals shedding Chlamydia at the respective days after genital challenge. day 80 after chlamydial challenge compared to animals immunized with BMDCs pulsed with HEL + CpG (mock immunized, $50 \%$ bilateral). BMDCs pulsed with UV-EB + CpG immunization induced comparable levels of protection against development of hydrosalpinx (33\% bilateral) to those pulsed with rCPAF + CpG, following chlamydial challenge (Figure 6A).

Gross oviduct cross-sectional diameter also was measured as a quantitative indicator of the degree of hydrosalpinx. As shown in Figure 6B, animals immunized by BMDCs pulsed with $\mathrm{HEL}+\mathrm{CpG}$ (mock immunized) displayed a high degree of oviduct dilatation $(2 \pm 0.39 \mathrm{~mm})$ on day 80 after chlamydial challenge. In contrast, animals vaccinated with BMDCs pulsed with $\mathrm{rCPAF}+\mathrm{CpG}$ displayed significantly reduced dilatation of oviducts $(0.66 \pm 0.21 \mathrm{~mm})$. Animals vaccinated with UV-EB pulsed BMDCs also displayed significant reductions $(1.21 \pm 0.26 \mathrm{~mm})$ in dilatation score.

The presence of inflammatory cellular infiltrates in the genital tract tissues was scored by microscopy (Figure 6C). Mice immunized with BMDCs pulsed with $\mathrm{rCPAF}+\mathrm{CpG}$ or UV$\mathrm{EB}+\mathrm{CpG}$ displayed significant $(p \leq 0.05)$ reductions in mononuclear cells $(0.92 \pm 0.25$ and $1.08 \pm 0.20$, respectively) and plasma cells $(0.42 \pm 0.15$ and $0.33 \pm 0.11$ respectively), compared to those immunized with BMDCs pulsed with HEL + CpG (mononuclear cells $2.1 \pm 0.25$, plasma cells $1.42 \pm 0.35$ ). These results indicate that vaccination with BMDCs pulsed with $\mathrm{rCPAF}+\mathrm{CpG}$

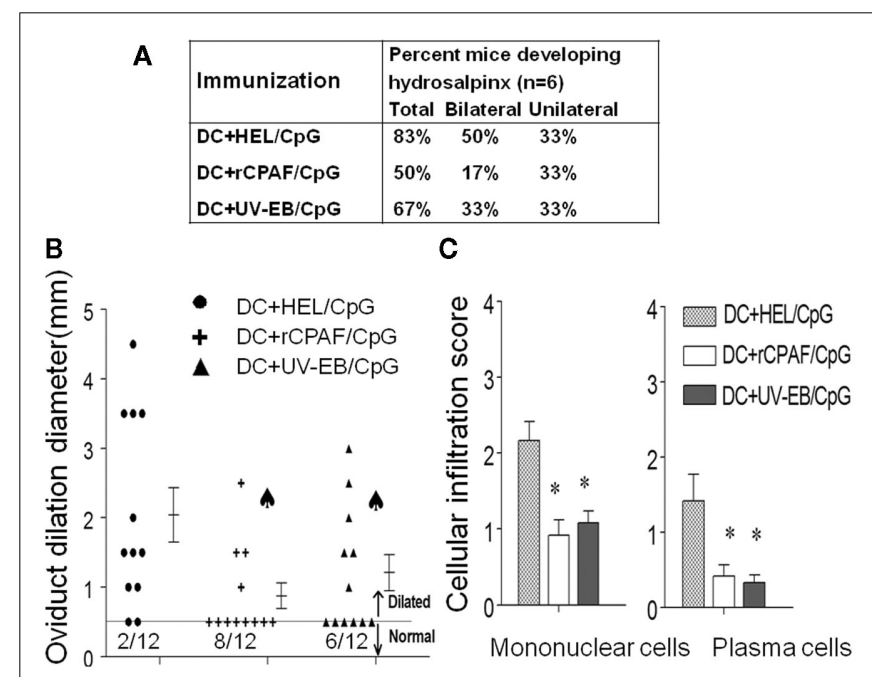

FIGURE 6 | The upper genital tract pathology was measured on day 80 after challenge. (A) Percentage of mice developing hydrosalpinx in different immunization groups after genital chlamydial challenge. (B) The oviduct dilation after vaccination and chlamydial challenge. Groups (six mice per group) were immunized s.c. with $\mathrm{HEL}+\mathrm{CpG}, \mathrm{rCPAF}+\mathrm{CpG}$, or UV-EB $+C p G$ on day 0 , and booster immunizations were given on day 14 . One month after the final immunization, mice were challenged i.vag. with $5 \times 10^{4}$ IFU of C. muridarum. On day 80 after challenge, oviduct dilatation was measured. Each individual marker represents one oviduct, and the mean \pm SEM of oviduct diameter per group of mice also is shown. (*) Significant ( $p<0.05$, chi-square test) difference in oviduct diameter between the indicated group and $\mathrm{HEL}+\mathrm{CpG}$-pulsed group, respectively. (C) On day 80 after challenge, infiltration of mononuclear cells and plasma cells were scored. The means \pm SEM of the scores per group are shown. (*) Significant differences ( $p<0.05$; Kruskal-Wallis one-way ANOVA on ranks) between the indicated groups and $\mathrm{HEL}+\mathrm{CpG}$-pulsed group are shown. 
induces robust protection against oviduct pathology and reduces inflammatory cellular infiltration.

\section{DISCUSSION}

Dendritic cells are central to induction of immunity to chlamydial genital tract infection, and potent immunity against genital tract reinfection, comparable to that induced by prior live chlamydial infection and has been achieved in the mouse model by immunization with DCs pulsed ex vivo with whole inactivated organisms (Su et al., 1998; Rey-Ladino et al., 2005). Thus, DC-based vaccination and immunotherapy provide a promising new tool for development of an effective Chlamydia vaccine. There have been positive efforts to improve DC-based vaccination approaches for cancer immunotherapy in the last decade. Several clinical trials of human DC-based vaccines are generating encouraging preliminary results demonstrating that there is an induction of tumorspecific immune responses and tumor regression (Santegoets et al., 2008; Le et al., 2010). These clinical DC-based vaccinations have been proved to be feasible and safe, although continued optimization is still required for broader cancer immunotherapy and potential applications against infectious diseases.

The present study shows that BMDCs pulsed ex vivo with $\mathrm{rCPAF}+\mathrm{CpG}$ are effective in inducing optimal surface activation markers and functional maturation of DCs, including upregulated expression of CD86, CD80, CD40, MHC II; enhanced production of IL-12, but not IL-10 and IL-4, and an increased ability to process and present CPAF antigens to Chlamydia-specific $\mathrm{CD}^{+}{ }^{+} \mathrm{T}$ cells. Moreover, protective immunity against genital infection can be achieved by subcutaneous immunization of mice with BMDCs pulsed ex vivo with $\mathrm{rCPAF}+\mathrm{CpG}$. These mice also displayed complete vaginal chlamydial clearance by day 18 postchallenge and a reduced incidence (50\%) of hydrosalpinx and histopathology, compared to clearance on day 30 and hydrosalpinx in $83 \%$ mice in HEL + CpG-BMDCs immunized mice. These results demonstrate that BMDCs pulsed with a recombinant chlamydial antigen may be used to induce robust anti-Chlamydia protective immunity at the genital mucosa.

Subcutaneous immunization with BMDCs pulsed ex vivo with $\mathrm{rCPAF}+\mathrm{CpG}$ also induced robust antibody and cell mediated antigen-specific Th1 type responses and IFN- $\gamma$ production, which resulted in the significant enhancement of genital chlamydial clearance and reduction of oviduct pathology. Although DC-based vaccines can efficiently present antigen to $\mathrm{B}$ cells and $\mathrm{CD}^{+} \mathrm{T}$ cells, the activation of $\mathrm{CD}^{+}{ }^{+} \mathrm{T}$ cells by DCs can exert helper functions to enhance efficient antibody production, production of high-affinity antibodies through somatic hypermutation, and class-switching of antibodies (Tamura and Chiba, 2009). However, there was no evidence of resistance to initial infection, or a further enhancement in protective immunity compared to that induced by intranasal vaccination with soluble rCPAF + CpG (Cong et al., 2007) or rCPAF + IL-12 (Murthy et al., 2007) as demonstrated in

\section{REFERENCES}

Allam, J. P., Bieber, T., and Novak, N. (2009). Dendritic cells as potential targets for mucosal immunotherapy. Curr. Opin. Allergy Clin. Immunol. 9, $554-557$.
Brunham, R. C., and Rey-Ladino, J. (2005). Immunology of Chlamydia infection: implications for a Chlamydia trachomatis vaccine. Nat. Rev. Immunol. 5, 149-161.

our previous published studies. These results may suggest that the degree of priming obtained by DC vaccination and/or subsequent activation of $\mathrm{CD}^{+}{ }^{+} \mathrm{T}$ cells may be comparable to that obtained following vaccination with soluble antigen. The lack of resistance to initial infection confirms and extends our prior findings that anti-CPAF antibodies are not capable of neutralizing chlamydial infectivity (Li et al., 2010). We hypothesize that this results directly from the fact that CPAF is a late-phase protein, produced only during the $\mathrm{RB}$ phase, and not present in the infectious $\mathrm{EB}$ phase of the chlamydial organism. Additionally, DC immunization may induce robust antigen-specific IFN- $\gamma$ responses, but did not result in superior protective immunity when compared to soluble rCPAF immunization. This may suggest that cell mediated immunity is only capable of inducing enhanced and complete clearance around days 15-18 after genital C. muridarum challenge. This also is supported by clearance of infection in a similar time period following immunization with BMDCs pulsed with inactivated whole chlamydial organisms. However, it cannot be excluded that BMDC immunization administered intravenously as in previous reports (Su et al., 1998; He et al., 2005), instead of subcutaneously as in this study, may have resulted in chlamydial clearance at earlier time periods. In the previous reports, the BMDC immunization was delivered via the retro-orbital plexus (Su et al., 1998), a method that has been considered invasive and less favorable in recent years. Given the promising potential of CPAF in inducing antichlamydial protective immunity, it may be worthwhile to evaluate intravenous immunization with rCPAF-pulsed BMDCs via the tail vein in future studies.

Collectively, BMDCs pulsed ex vivo with rCPAF plus $\mathrm{CpG}$ induced enhancements in genital chlamydial clearance and reduction of oviduct pathology comparable to our past studies with soluble rCPAF + CpG intranasal vaccination (Murthy et al., 2007; Li et al., 2010). These results confirm and extend our previous reports on the efficacy of CPAF immunization in reducing Chlamydiainduced upper genital tract pathological sequelae. They also suggest that inducing chlamydial clearance at earlier time periods may require the inclusion of a chlamydial surface antigen, such as native MOMP which has been shown to induce antibodies that neutralize chlamydial infectivity (Pal et al., 2008; Kari et al., 2009), into a combination vaccine to achieve optimal anti-chlamydial protective immunity. Such a combination would display the benefit of MOMP mediated neutralizing antibody effects against specific serovars with partial resistance to infection resulting in reduced transmission, and cell mediated immunity induced by CPAF against multiple serovars and species of Chlamydia, resulting in reduction of oviduct pathological sequelae.

\section{ACKNOWLEDGMENTS}

This work has been supported by the National Institutes of Health grant 1RO1AI074860 and the Army Research Office of the Department of Defense under Contract No. W911NF-11-1-0136.

Chen, D., Lei, L., Lu, C., Galaleldeen, A., Hart, P. J., and Zhong, G. (2010). Characterization of Pgp3, a Chlamydia trachomatis plasmidencoded immunodominant antigen. J. Bacteriol. 192, 6017-6024.
Cong, Y., Jupelli, M., Guentzel, M. N., Zhong, G., Murthy, A. K., and Arulanandam, B. P. (2007). Intranasal immunization with chlamydial protease-like activity factor and CpG deoxynucleotides enhances 
protective immunity against genital Chlamydia muridarum infection. Vaccine 25, 3773-3780.

Dong, F., Sharma, J., Xiao, Y., Zhong, Y., and Zhong, G. (2004). Intramolecular dimerization is required for the Chlamydia-secreted protease CPAF to degrade host transcriptional factors. Infect. Immun. 72, 3869-3875.

Eaton, K. A., Benson, L. H., Haeger, J., and Gray, B. M. (2006). Role of transcription factor T-bet expression by $\mathrm{CD} 4^{+}$cells in gastritis due to Helicobacter pylori in mice. Infect. Immun. 74, 4673-4684.

Eko, F, O., Okenu, D. N., Singh, U. P., He, Q., Black, C., and Igietseme, J. U. (2011). Evaluation of a broadly protective Chlamydia-cholera combination vaccine candidate. Vaccine 29, 3802-3810.

Gottlieb, S. L., Martin, D. H., Xu, F., Byrne, G. I., and Brunham, R. C. (2010). Summary: the natural history and immunobiology of Chlamydia trachomatis genital infection and implications for Chlamydia control. J. Infect. Dis. 201(Suppl. 2), S190-S204.

Hafner, L. M., and McNeilly, C. (2008). Vaccines for Chlamydia infections of the female genital tract. Future Microbiol. 3, 67-77.

He, Q., Moore, T. T., Eko, F. O., Lyn, D., Ananaba, G. A., Martin, A., Singh, S., James, L., Stiles, J., Black, C. M., and Igietseme, J. U. (2005). Molecular basis for the potency of IL-10deficient dendritic cells as a highly efficient APC system for activating Th1 response. J. Immunol. 174, 4860-4869.

Huang, J., Wang, M. D., Lenz, S., Gao, D., and Kaltenboeck, B. (1999). IL12 administered during Chlamydia psittaci lung infection in mice confers immediate and long-term protection and reduces macrophage inflammatory protein-2 level and neutrophil infiltration in lung tissue. J. Immunol. 162, 2217-2226.

Kari, L., Whitmire, W. M., Crane, D. D., Reveneau, N., Carlson, J. H., Goheen, M. M., Peterson, E. M., Pal, S., de la Maza, L. M., and Caldwell, H. D. (2009). Chlamydia trachomatis native major outer membrane protein induces partial protection in nonhuman primates: implication for a trachoma transmissionblocking vaccine. J. Immunol. 182, 8063-8070.

Le, D. T., Pardoll, D. M., and Jaffee, E. M. (2010). Cellular vaccine approaches. Cancer J. 16, 304-310.
Li, W., Guentzel, M. N., Seshu, J., Zhong, G., Murthy, A. K., and Arulanandam, B. P. (2007). Induction of cross-serovar protection against genital chlamydial infection by a targeted multisubunit vaccination approach. Clin. Vaccine Immunol. 14, 1537-1544.

Li, W., Murthy, A. K., Guentzel, M. N., Chambers, J. P., Forsthuber, T. G., Seshu, J., Zhong, G., and Arulanandam, B. P. (2010). Immunization with a combination of integral chlamydial antigens and a defined secreted protein induces robust immunity against genital chlamydial challenge. Infect. Immun. 78, 3942-3949.

Li, W., Murthy, A. K., Guentzel, M. N., Seshu, J., Forsthuber, T. G., Zhong, G., and Arulanandam, B. P. (2008). Antigen-specific CD4 ${ }^{+} \mathrm{T}$ cells produce sufficient IFN-gamma to mediate robust protective immunity against genital Chlamydia muridarum infection. J. Immunol. 180, 3375-3382.

Lu, H., and Zhong, G. (1999). Interleukin-12 production is required for chlamydial antigenpulsed dendritic cells to induce protection against live Chlamydia trachomatis infection. Infect. Immun. 67, 1763-1769.

Morrison, R. P., and Caldwell, H. D. (2002). Immunity to murine chlamydial genital infection. Infect. Immun. 70, 2741-2751.

Murthy, A. K., Chambers, J. P., Meier, P. A., Zhong, G., and Arulanandam, B. P. (2007). Intranasal vaccination with a secreted chlamydial protein enhances resolution of genital Chlamydia muridarum infection, protects against oviduct pathology and is highly dependent upon endogenous IFN-\{gamma\} production. Infect. Immun. 75, 666-676.

Murthy, A. K., Li, W., Guentzel, M. N., Zhong, G., and Arulanandam, B. P. (2011). Vaccination with the defined chlamydial secreted protein CPAF induces robust protection against female infertility following repeated genital chlamydial challenge. Vaccine 29, 2519-2522.

Olsen, A. W., Theisen, M., Christensen, D., Follmann, F., and Andersen, P. (2010). Protection against Chlamydia promoted by a subunit vaccine $(\mathrm{CTH} 1)$ compared with a primary intranasal infection in a mouse genital challenge model. PLoS ONE 5, e10768. doi:10.1371/journal.pone. 0010768
Pal, S., Bravo, J., Peterson, E. M. and de la Maza, L. M. (2008). Protection of wild-type and severe combined immunodeficiency mice against an intranasal challenge by passive immunization with monoclonal antibodies to the Chlamydia trachomatis mouse pneumonitis major outer membrane protein. Infect. Immun. 76, 5581-5587.

Pal, S., Peterson, E. M., and de la Maza, L. M. (2005). Vaccination with the Chlamydia trachomatis major outer membrane protein can elicit an immune response as protective as that resulting from inoculation with live bacteria. Infect. Immun. 73, 8153-8160.

Perry, L. L., Feilzer, K., and Caldwell, H. D. (1997). Immunity to Chlamydia trachomatis is mediated by $\mathrm{T}$ helper 1 cells through IFN-gammadependent and -independent pathways. J. Immunol. 158, 3344-3352.

Rank, G. R. (1999). Chlamydia Intracellular Biology, Pathogenesis and Immunity. Washington, DC: ASM Press, 239-296.

Rey-Ladino, J., Koochesfahani, K. M., Zaharik, M. L., Shen, C., and Brunham, R. C. (2005). A live and inactivated Chlamydia trachomatis mouse pneumonitis strain induces the maturation of dendritic cells that are phenotypically and immunologically distinct. Infect. Immun. 73, 1568-1577.

Santegoets, S. J., van den Eertwegh, A. J., van de Loosdrecht, A. A., Scheper, R. J., and de Gruijl, T. D. (2008). Human dendritic cell line models for DC differentiation and clinical DC vaccination studies. J. Leukoc. Biol. 84, 1364-1373.

Siegemund, S., and Alber, G. (2008). Cryptococcus neoformans activates bone marrow-derived conventional dendritic cells rather than plasmacytoid dendritic cells and down-regulates macrophages. FEMS Immunol. Med. Microbiol. 52, 417-427.

Su, H., Messer, R., Whitmire, W., Fischer, E., Portis, J. C., and Caldwell, H. D. (1998). Vaccination against chlamydial genital tract infection after immunization with dendritic cells pulsed ex vivo with nonviable Chlamydiae. J. Exp. Med. 188 809-8018.

Sun, G., Pal, S., Weiland, J., Peterson, E. M., and de la Maza, L. M. (2009). Protection against an intranasal challenge by vaccines formulated with native and recombinant preparations of the Chlamydia trachomatis major outer membrane protein. Vaccine 27, 5020-5025.

Svensson, M., Stockinger, B., and Wick, M. J. (1997). Bone marrow-derived dendritic cells can process bacteria for MHC-I and MHC-II presentation to $\mathrm{T}$ cells. J. Immunol. 158, 4229-4236.

Tamura, T., and Chiba, J. (2009). Production of antibodies against multipass membrane proteins expressed in human tumor cells using dendritic cell immunization. J. Biomed. Biotechnol. 2009, 673098.

Yu, H., Jiang, X., Shen, C., Karunakaran, K. P., and Brunham, R. C. (2009). Novel Chlamydia muridarum $\mathrm{T}$ cell antigens induce protective immunity against lung and genital tract infection in murine models. J. Immunol. 182, 1602-1608.

Zhang, D., Yang, X., Lu, H., Zhong, G., and Brunham, R. C. (1999). Immunity to Chlamydia trachomatis mouse pneumonitis induced by vaccination with live organisms correlates with early granulocytemacrophage colony-stimulating factor and interleukin-12 production and with dendritic cell-like maturation. Infect. Immun. 67, 1606-1613.

Conflict of Interest Statement: The authors declare that the research was conducted in the absence of any commercial or financial relationships that could be construed as a potential conflict of interest.

Received: 05 October 2011; paper pending published: 01 November 2011; accepted: 23 November 2011; published online: 08 December 2011.

Citation: Li W, Murthy AK, Chaganty BKR, Guentzel MN, Seshu J, Chambers JP, Zhong $G$ and Arulanandam $B P$ (2011) Immunization with dendritic cells pulsed ex vivo with recombinant chlamydial protease-like activity factor induces protective immunity against genital Chlamydia muridarum challenge. Front. Immun. 2:73. doi: 10.3389/fimmu.2011.00073

This article was submitted to Frontiers in Microbial Immunology, a specialty of Frontiers in Immunology.

Copyright () 2011 Li, Murthy, Chaganty, Guentzel, Seshu, Chambers, Zhong and Arulanandam. This is an open-access article distributed under the terms of the Creative Commons Attribution Non Commercial License, which permits noncommercial use, distribution, and reproduction in other forums, provided the original authors and source are credited. 